\title{
SEVERAL EXPLICIT AND RECURRENT FORMULAS FOR DETERMINANTS OF TRIDIAGONAL MATRICES VIA GENERALIZED CONTINUED FRACTIONS
}

\author{
FENG QI, WEN WANG, BAI-NI GUO*, AND DONGKYU LIM*
}

\begin{abstract}
In the paper, by the aid of mathematical induction and some properties of determinants, the authors present several explicit and recurrent formulas of evaluations for determinants of general tridiagonal matrices in terms of finite generalized continued fractions and apply these newly-established formulas to evaluations for determinants of the Sylvester matrix and two Sylvester type matrices.
\end{abstract}

\section{Contents}

1. Introduction 233

2. $\quad$ Explicit and recurrent formulas for $\left|P_{n}\right| \mid \quad 235$

\begin{tabular}{|lll|}
\hline 3. & Explicit and recurrent formulas for $\left|D_{n}\right| \mid$ & 237
\end{tabular}

$\begin{array}{lll}4 . \quad \text { Discussions } & 240\end{array}$

\begin{tabular}{ll}
\hline Acknowledgements & 247
\end{tabular}

$\begin{array}{ll}\text { Funding } & 247\end{array}$

Conflict of interest 247

References 247

\section{INTRODUCTION}

A finite generalized continued fraction is of the form

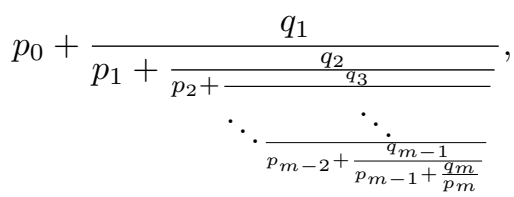

2010 Mathematics Subject Classification. Primary 15A15; Secondary 11C20, 15B05, 15B99, 65F40.

Key words and phrases. determinant; tridiagonal matrix; induction; explicit formula; recurrent formula; finite generalized continued fraction; Sylvester matrix; Sylvester type matrix.

*Corresponding author.

Please cite this article as "Feng Qi, Wen Wang, Bai-Ni Guo, and Dongkyu Lim, Several explicit and recurrent formulas for determinants of tridiagonal matrices via generalized continued fractions, Chapter 15 in Zakia Hammouch, Hemen Dutta, Said Melliani, and Michael Ruzhansky, Nonlinear Analysis: Problems, Applications and Computational Methods, SM2A 2019, pp. 233-248; Springer Book Series Lecture Notes in Networks and Systems, Volume 168, 2021; available online at https://doi.org/10.1007/978-3-030-62299-2_15."

This paper was typeset using $\mathcal{A} \mathcal{M S}$-LATEX. 
where $p_{0}, p_{1}, \ldots, p_{m}$ and $q_{1}, q_{2}, \ldots, q_{m}$ can be any complex numbers or functions. It can also be written equivalently as

$$
p_{0}+\stackrel{m}{K}_{\ell=1}^{m} \frac{q_{\ell}}{p_{\ell}}=p_{0}+\sum_{\ell=1}^{m} \frac{q_{\ell} \mid}{\mid p_{\ell}}=p_{0}+\frac{q_{1}}{p_{1}+} \frac{q_{2}}{p_{2}+} \cdots \frac{q_{m-1}}{p_{m-1}+} \frac{q_{m}}{p_{m}} .
$$

In this paper, we will use the second compact form above. For more information on the theory of continued fractions, please refer to the papers [7, 14] and closely related references therein.

In general, a tridiagonal matrix of order $n$ is defined for $n \in \mathbb{N}$ by

$$
D_{n}=\left(e_{i, j}\right)_{1 \leq i, j \leq n}=\left(\begin{array}{ccccccccc}
\alpha_{1} & \beta_{1} & 0 & 0 & \cdots & 0 & 0 & 0 & 0 \\
\gamma_{1} & \alpha_{2} & \beta_{2} & 0 & \cdots & 0 & 0 & 0 & 0 \\
0 & \gamma_{2} & \alpha_{3} & \beta_{3} & \cdots & 0 & 0 & 0 & 0 \\
0 & 0 & \gamma_{3} & \alpha_{4} & \cdots & 0 & 0 & 0 & 0 \\
\vdots & \vdots & \vdots & \vdots & \ddots & \vdots & \vdots & \vdots & \vdots \\
0 & 0 & 0 & 0 & \cdots & \alpha_{n-3} & \beta_{n-3} & 0 & 0 \\
0 & 0 & 0 & 0 & \cdots & \gamma_{n-3} & \alpha_{n-2} & \beta_{n-2} & 0 \\
0 & 0 & 0 & 0 & \cdots & 0 & \gamma_{n-2} & \alpha_{n-1} & \beta_{n-1} \\
0 & 0 & 0 & 0 & \cdots & 0 & 0 & \gamma_{n-1} & \alpha_{n}
\end{array}\right),
$$

where

$$
e_{i, j}= \begin{cases}\alpha_{i}, & 1 \leq i=j \leq n \\ \beta_{i}, & 1 \leq i=j-1 \leq n-1 ; \\ \gamma_{j}, & 1 \leq j=i-1 \leq n-1 ; \\ 0, & \text { otherwise. }\end{cases}
$$

In the papers 15, 16, 18, the determinant $\left|D_{n}\right|$ and some special cases were discussed, computed, and applied to several problems in analytic combinatorics and analytic number theory. In the papers [2, 5, 6, 9, 15, 16, 18, there are some computation of the inverse and determinant of the general tridiagonal matrix $D_{n}$. For more information about this topic, please refer to the papers [4, 8, 12, 13] and closely related references therein.

Let $n \geq 2$ and

$$
P_{n}=\left(p_{i, j}\right)_{1 \leq i, j \leq n}=\left(\begin{array}{cccccccccc}
a_{1} & c_{1} & 0 & 0 & \cdots & 0 & 0 & 0 & 0 \\
a_{2} & b_{2} & c_{2} & 0 & \cdots & 0 & 0 & 0 & 0 \\
a_{3} & 0 & b_{3} & c_{3} & \cdots & 0 & 0 & 0 & 0 \\
a_{4} & 0 & 0 & b_{4} & \cdots & 0 & 0 & 0 & 0 \\
\vdots & \vdots & \vdots & \vdots & \ddots & \vdots & \vdots & \vdots & \vdots \\
a_{n-3} & 0 & 0 & 0 & \cdots & b_{n-3} & c_{n-3} & 0 & 0 \\
a_{n-2} & 0 & 0 & 0 & \cdots & 0 & b_{n-2} & c_{n-2} & 0 \\
a_{n-1} & 0 & 0 & 0 & \cdots & 0 & 0 & b_{n-1} & c_{n-1} \\
a_{n} & 0 & 0 & 0 & \cdots & 0 & 0 & 0 & b_{n}
\end{array}\right),
$$


where

$$
p_{i, j}= \begin{cases}a_{i}, & 1 \leq i \leq n, j=1 \\ b_{i}, & 2 \leq i=j \leq n \\ c_{i}, & 1 \leq i=j-1 \leq n-1 ; \\ 0, & \text { otherwise. }\end{cases}
$$

In this paper, by the help of mathematical induction and some properties of determinants, we will present several explicit and recurrent formulas for evaluations of two determinants $\left|P_{n}\right|$ and $\left|D_{n}\right|$ and will apply these newly-established formulas to evaluations for determinants of the Sylvester matrix and two Sylvester type matrices.

\section{Explicit AND RECURRENT FORMulas For $\left|P_{n}\right|$}

Right now we start off to present explicit and recurrent formulas for $\left|P_{n}\right|$.

Theorem 2.1. Let $n \geq 2$ and $b_{k} \neq 0$ for $2 \leq k \leq n$. Then the determinant $\left|P_{n}\right|$ can be computed recurrently by

$$
\left|P_{n}\right|=\lambda_{1, n} \prod_{k=2}^{n} b_{k}
$$

where

and $\lambda_{n, n}=a_{n}$

$$
\lambda_{k, n}=a_{k}-\frac{c_{k}}{b_{k+1}} \lambda_{k+1, n}, \quad 1 \leq k \leq n-1
$$

Proof. When $n=2$, it is easy to see that

$$
\left|P_{2}\right|=\left|\begin{array}{ll}
a_{1} & c_{1} \\
a_{2} & b_{2}
\end{array}\right|=a_{1} b_{2}-a_{2} c_{1}
$$

and

$$
\lambda_{1,2} \prod_{k=2}^{2} b_{k}=\lambda_{1,2} b_{2}=\left(a_{1}-\frac{c_{1} \lambda_{2,2}}{b_{2}}\right) b_{2}=\left(a_{1}-\frac{c_{1} a_{2}}{b_{2}}\right) b_{2}=a_{1} b_{2}-a_{2} c_{1}=\left|P_{2}\right| .
$$

This means that the formula 2.1) is valid for $n=2$.

Assume that the formula 2.1 validates for $n=m-1$, equivalently speaking,

$$
\left|P_{m-1}\right|=\lambda_{1, m-1} \prod_{k=2}^{m-1} b_{k}
$$


When $n=m$, expanding the determinant $\left|P_{m}\right|$ according to the first rank and utilizing the assumption for $n=m-1$ give

$$
\begin{gathered}
\left|P_{m}\right|=a_{1} \prod_{k=2}^{m} b_{k}-c_{1}\left|\begin{array}{cccccccc}
a_{2} & c_{2} & 0 & \cdots & 0 & 0 & 0 & 0 \\
a_{3} & b_{3} & c_{3} & \cdots & 0 & 0 & 0 & 0 \\
a_{4} & 0 & b_{4} & \cdots & 0 & 0 & 0 & 0 \\
\vdots & \vdots & \vdots & \ddots & \vdots & \vdots & \vdots & \vdots \\
a_{m-3} & 0 & 0 & \cdots & b_{m-3} & c_{m-3} & 0 & 0 \\
a_{m-2} & 0 & 0 & \cdots & 0 & b_{m-2} & c_{m-2} & 0 \\
a_{m-1} & 0 & 0 & \cdots & 0 & 0 & b_{m-1} & c_{m-1} \\
a_{m} & 0 & 0 & \cdots & 0 & 0 & 0 & b_{m}
\end{array}\right| \\
=a_{1} \prod_{k=2}^{m} b_{k}-c_{1} \lambda_{2, m} \prod_{k=3}^{m} b_{k}=\left(a_{1}-\frac{c_{1}}{b_{2}} \lambda_{2, m}\right) \prod_{k=2}^{m} b_{k}=\lambda_{1, m} \prod_{k=2}^{m} b_{k} .
\end{gathered}
$$

By mathematical induction, we derive the formula 2.1. The proof of Theorem 2.1 is complete.

Theorem 2.2. For $n \geq 2$, the determinant $\left|P_{n}\right|$ can be computed explicitly by

$$
\left|P_{n}\right|=a_{1} \prod_{k=2}^{n} b_{k}-\sum_{k=2}^{n}(-1)^{k}\left(\prod_{\ell=1}^{k-1} c_{\ell} \prod_{m=k+1}^{n} b_{m}\right) a_{k} .
$$

Proof. From the recurrent relation 2.2., it follows that

$$
\begin{aligned}
\lambda_{1, n}= & a_{1}-\frac{c_{1}}{b_{2}} \lambda_{2, n} \\
= & a_{1}-\frac{c_{1}}{b_{2}}\left(a_{2}-\frac{c_{2}}{b_{3}} \lambda_{3, n}\right) \\
= & a_{1}-\frac{c_{1}}{b_{2}}\left[a_{2}-\frac{c_{2}}{b_{3}}\left(a_{3}-\frac{c_{3}}{b_{4}} \lambda_{4, n}\right)\right] \\
= & \cdots \\
= & a_{1}-\frac{c_{1}}{b_{2}}\left[a_{2}-\frac{c_{2}}{b_{3}}\left(a_{3}-\frac{c_{3}}{b_{4}}\left[a_{4}-\cdots-\frac{c_{\ell-1}}{b_{\ell}}\left(a_{\ell}-\frac{c_{\ell}}{b_{\ell+1}} \lambda_{\ell+1, n}\right)\right]\right)\right] \\
= & \cdots \\
= & a_{1}-\frac{c_{1}}{b_{2}}\left[a_{2}-\frac{c_{2}}{b_{3}}\left(a_{3}-\frac{c_{3}}{b_{4}}\left[a_{4}-\cdots-\frac{c_{\ell-1}}{b_{\ell}}\left(a_{\ell}-\cdots\right.\right.\right.\right. \\
& \left.\left.\left.\left.-\frac{c_{n-3}}{b_{n-2}}\left[a_{n-2}-\frac{c_{n-2}}{b_{n-1}}\left(a_{n-1}-\frac{c_{n-1}}{b_{n}} \lambda_{n, n}\right)\right]\right)\right]\right)\right] \\
= & a_{1}-\frac{c_{1}}{b_{2}}\left[a_{2}-\frac{c_{2}}{b_{3}}\left(a_{3}-\frac{c_{3}}{b_{4}}\left[a_{4}-\cdots-\frac{c_{\ell-1}}{b_{\ell}}\left(a_{\ell}-\cdots\right.\right.\right.\right. \\
& \left.\left.\left.\left.-\frac{c_{n-3}}{b_{n-2}}\left[a_{n-2}-\frac{c_{n-2}}{b_{n-1}}\left(a_{n-1}-\frac{c_{n-1}}{b_{n}} a_{n}\right)\right]\right)\right]\right)\right]
\end{aligned}
$$




$$
\begin{aligned}
= & a_{1}-\frac{c_{1}}{b_{2}}\left(a_{2}-\frac{c_{2}}{b_{3}}\left[a_{3}-\frac{c_{3}}{b_{4}}\left(a_{4}-\cdots-\frac{c_{\ell-1}}{b_{\ell}}\left[a_{\ell}-\cdots\right.\right.\right.\right. \\
& \left.\left.\left.\left.-\frac{c_{n-3}}{b_{n-2}}\left(a_{n-2}-\frac{c_{n-2}}{b_{n-1}} a_{n-1}+\frac{c_{n-2} c_{n-1}}{b_{n-1} b_{n}} a_{n}\right)\right]\right)\right]\right) \\
= & a_{1}-\frac{c_{1}}{b_{2}}\left(a_{2}-\frac{c_{2}}{b_{3}}\left[a_{3}-\frac{c_{3}}{b_{4}}\left(a_{4}-\cdots-\frac{c_{\ell-1}}{b_{\ell}}\left[a_{\ell}-\cdots\right.\right.\right.\right. \\
& \left.\left.\left.\left.-\left(\frac{c_{n-3}}{b_{n-2}} a_{n-2}-\frac{c_{n-3} c_{n-2}}{b_{n-2} b_{n-1}} a_{n-1}+\frac{c_{n-3} c_{n-2} c_{n-1}}{b_{n-2} b_{n-1} b_{n}} a_{n}\right)\right]\right)\right]\right) \\
= & \cdots \\
= & a_{1}-\sum_{k=2}^{n}(-1)^{k}\left(\prod_{\ell=2}^{k} \frac{c_{\ell-1}}{b_{\ell}}\right) a_{k}
\end{aligned}
$$

for $n \geq 2$. Substituting this result into 2.1) and simplifying lead to 2.3. The proof of Theorem 2.2 is complete.

Remark 2.1. Applying $a_{k}=k, b_{k}=k$, and $c_{k}=k$ to the explicit formula 2.3) in Theorem 2.2 reveals

$$
\left|\begin{array}{ccccccccc}
1 & 1 & 0 & 0 & \cdots & 0 & 0 & 0 & 0 \\
2 & 2 & 2 & 0 & \cdots & 0 & 0 & 0 & 0 \\
3 & 0 & 3 & 3 & \cdots & 0 & 0 & 0 & 0 \\
4 & 0 & 0 & 4 & \cdots & 0 & 0 & 0 & 0 \\
\vdots & \vdots & \vdots & \vdots & \ddots & \vdots & \vdots & \vdots & \vdots \\
n-3 & 0 & 0 & 0 & \cdots & n-3 & n-3 & 0 & 0 \\
n-2 & 0 & 0 & 0 & \cdots & 0 & n-2 & n-2 & 0 \\
n-1 & 0 & 0 & 0 & \cdots & 0 & 0 & n-1 & n-1 \\
n & 0 & 0 & 0 & \cdots & 0 & 0 & 0 & n
\end{array}\right|=\frac{1-(-1)^{n}}{2} n ! .
$$

\section{EXPLiCIT AND RECURRENT FORMULAS FOR $\left|D_{n}\right|$}

Now we are in a position to present explicit and recurrent formulas for $\left|D_{n}\right|$.

Theorem 3.1. For $n \in \mathbb{N}$, the determinant $\left|D_{n}\right|$ can be explicitly and recurrently computed by

$$
\begin{aligned}
\left|D_{n}\right|=\alpha_{1} \alpha_{2}+\left(\alpha_{1}-\beta_{1} \gamma_{1}\right) \prod_{m=3}^{n}[ & \left.\alpha_{m}+\prod_{\ell=1}^{m-2} \frac{\left(-\beta_{m-\ell} \gamma_{m-\ell}\right)}{\alpha_{m-\ell}}\right] \\
& -\sum_{k=3}^{n}\left[\prod_{\ell=1}^{k-1}\left(\beta_{\ell} \gamma_{\ell}\right)\right] \frac{\prod_{m=k+1}^{n}\left[\alpha_{m}+K_{\ell=1}^{m-2} \frac{\left(-\beta_{m-\ell} \gamma_{m-\ell}\right)}{\alpha_{m-\ell}}\right]}{\prod_{m=2}^{k-1}\left[\alpha_{m}+K_{\ell=1}^{m-2} \frac{\left(-\beta_{m-\ell} \gamma_{m-\ell}\right)}{\alpha_{m-\ell}}\right]}
\end{aligned}
$$

and

$$
\left|D_{n}\right|=\eta_{1, n}\left(\alpha_{2}+\prod_{k=3}^{n}\left[\alpha_{k}+\prod_{\ell=1}^{k-2} \frac{\left(-\beta_{k-\ell} \gamma_{k-\ell}\right)}{\alpha_{k-\ell}}\right]\right) \text {, }
$$


where $K_{\ell=q}^{p}$ for $p<q$ is understood to be zero,

$$
\begin{gathered}
\eta_{1, n}=-1-\frac{\beta_{1}}{\alpha_{2}} \eta_{2, n}, \quad \eta_{2, n}=\gamma_{1}-\frac{\beta_{2}}{\alpha_{3}-\frac{\beta_{2} \gamma_{2}}{\alpha_{2}}} \eta_{3, n}, \quad \eta_{3, n}=-\frac{\gamma_{1} \gamma_{2}}{\alpha_{2}}-\frac{\beta_{3}}{\alpha_{4}-\frac{\beta_{3} \gamma_{3}}{\alpha_{3}-\frac{\beta_{2} \gamma_{2}}{\alpha_{2}}}} \eta_{4, n}, \\
\eta_{k, n}=(-1)^{k} \frac{\prod_{\ell=1}^{k-1} \gamma_{\ell}}{\alpha_{2}+\prod_{\ell=3}^{k-1}\left[\alpha_{\ell}+K_{m=1}^{\ell-2} \frac{\left(-\beta_{\ell-m} \gamma_{\ell-\ell}\right)}{\alpha_{\ell-m}}\right]}-\frac{\beta_{k}}{\alpha_{k+1}+K_{\ell=1}^{k-1} \frac{\left(-\beta_{k-\ell+1} \gamma_{k-\ell+1}\right)}{\alpha_{k-\ell+1}}} \eta_{k+1, n}
\end{gathered}
$$

for $4 \leq k \leq n-1$, and

$$
\eta_{n, n}=(-1)^{n} \frac{\prod_{\ell=1}^{n-1} \gamma_{\ell}}{\alpha_{2}+\prod_{k=3}^{n-1}\left[\alpha_{k}+K_{\ell=1}^{k-2} \frac{\left(-\beta_{k-\ell} \gamma_{k-\ell}\right)}{\alpha_{k-\ell}}\right]} .
$$

Proof. The determinant $\left|D_{n}\right|$ of the tridiagonal matrix $D_{n}$ in 1.1 can be rewritten as

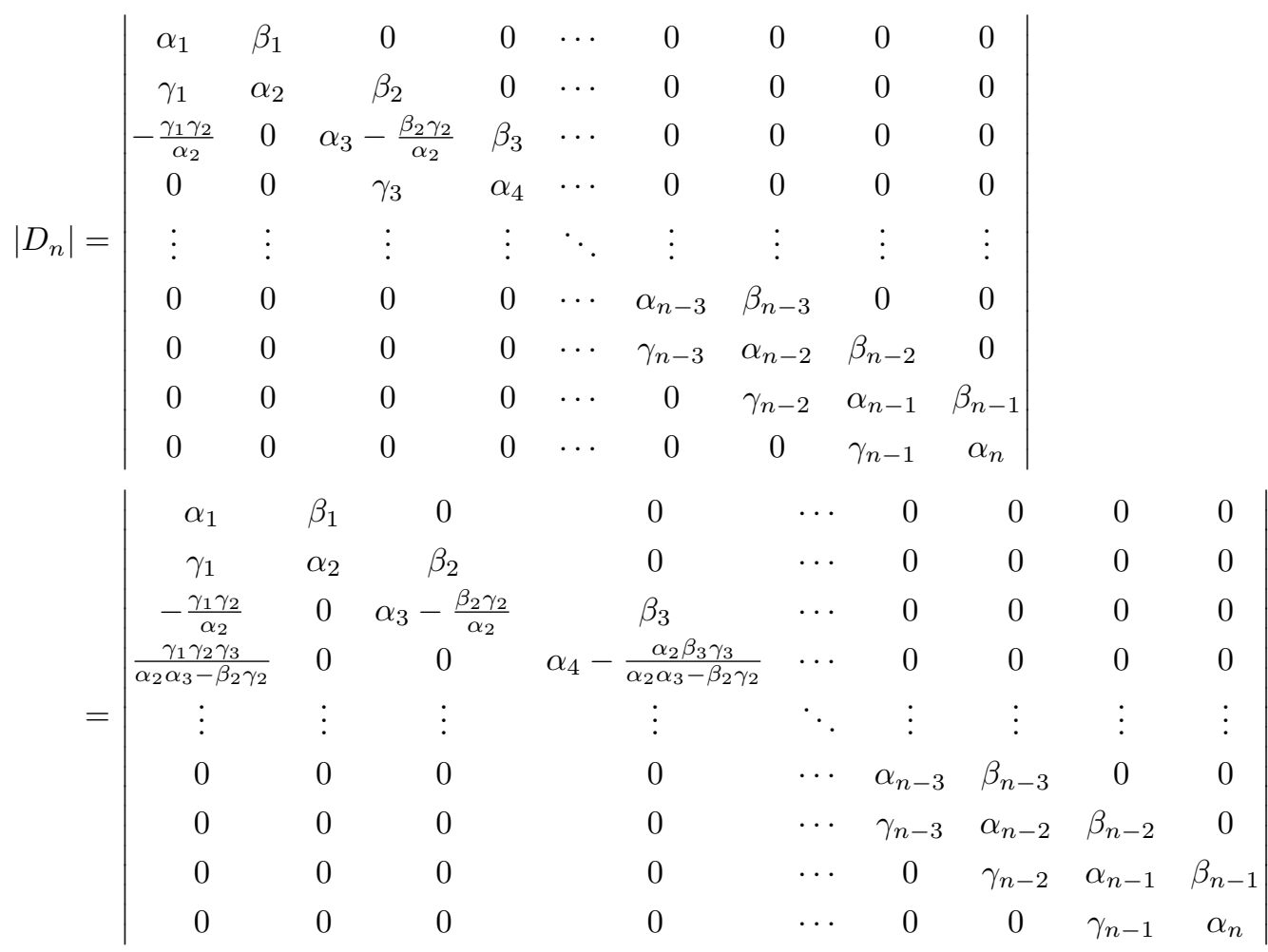

$$
\begin{aligned}
& =\cdots
\end{aligned}
$$




$$
=\left|\begin{array}{ccccccccc}
a_{1} & \beta_{1} & 0 & 0 & \cdots & 0 & 0 & 0 & 0 \\
a_{2} & b_{2} & \beta_{2} & 0 & \cdots & 0 & 0 & 0 & 0 \\
a_{3} & 0 & b_{3} & \beta_{3} & \cdots & 0 & 0 & 0 & 0 \\
a_{4} & 0 & 0 & b_{4} & \cdots & 0 & 0 & 0 & 0 \\
\vdots & \vdots & \vdots & \vdots & \ddots & \vdots & \vdots & \vdots & \vdots \\
a_{n-3} & 0 & 0 & 0 & \cdots & b_{n-3} & \beta_{n-3} & 0 & 0 \\
a_{n-2} & 0 & 0 & 0 & \cdots & 0 & b_{n-2} & \beta_{n-2} & 0 \\
a_{n-1} & 0 & 0 & 0 & \cdots & 0 & 0 & b_{n-1} & \beta_{n-1} \\
a_{n} & 0 & 0 & 0 & \cdots & 0 & 0 & 0 & b_{n}
\end{array}\right|,
$$

where

$$
\begin{gathered}
b_{2}=\alpha_{2}, \quad b_{3}=\alpha_{3}-\frac{\beta_{2} \gamma_{2}}{b_{2}}, \quad b_{4}=\alpha_{4}-\frac{\beta_{3} \gamma_{3}}{b_{3}}, \quad \ldots, \quad b_{n-3}=\alpha_{n-3}-\frac{\beta_{n-4} \gamma_{n-4}}{b_{n-4}}, \\
b_{n-2}=\alpha_{n-2}-\frac{\beta_{n-3} \gamma_{n-3}}{b_{n-3}}, \quad b_{n-1}=\alpha_{n-1}-\frac{\beta_{n-2} \gamma_{n-2}}{b_{n-2}}, \quad b_{n}=\alpha_{n}-\frac{\beta_{n-1} \gamma_{n-1}}{b_{n-1}}
\end{gathered}
$$

and

$$
\begin{gathered}
a_{1}=\alpha_{1}, \quad a_{2}=\gamma_{1}, \quad a_{3}=-\frac{\gamma_{2}}{b_{2}} a_{2}, \quad a_{4}=-\frac{\gamma_{3}}{b_{3}} a_{3}, \quad \ldots, \quad a_{n-3}=-\frac{\gamma_{n-4}}{b_{n-4}} a_{n-4}, \\
a_{n-2}=-\frac{\gamma_{n-3}}{b_{n-3}} a_{n-3}, \quad a_{n-1}=-\frac{\gamma_{n-2}}{b_{n-2}} a_{n-2}, \quad a_{n}=-\frac{\gamma_{n-1}}{b_{n-1}} a_{n-1} .
\end{gathered}
$$

The sequences $b_{k}$ and $a_{k}$ for $k \geq 3$ can be formulated by finite generalized continued fractions

$$
b_{k}=\alpha_{k}-\frac{\beta_{k-1} \gamma_{k-1}}{\alpha_{k-1}-\frac{\beta_{k-2} \gamma_{k-2}}{\alpha_{k-2}-\frac{\beta_{k-3} \gamma_{k-3}}{\alpha_{k-3}-\frac{\beta_{k-4} \gamma_{k-4}}{\cdot \frac{\ddots^{\prime}}{\alpha_{4}-\frac{\beta_{3} \gamma_{3}}{\alpha_{3}-\frac{\beta_{2} \gamma_{2}}{\alpha_{2}}}}}}}}=\alpha_{k}+\stackrel{k-2}{K} \frac{\left(-\beta_{k-\ell} \gamma_{k-\ell}\right)}{\alpha_{k-\ell}}
$$

and

Using 2.3 results in

$$
\begin{aligned}
\left|D_{n}\right|= & \left(b_{2}+\prod_{k=3}^{n} B_{k}\right) a_{1}-\left(\beta_{1} \prod_{m=3}^{n} B_{m}\right) a_{2}-\sum_{k=3}^{n}(-1)^{k}\left(\prod_{\ell=1}^{k-1} \beta_{\ell} \prod_{m=k+1}^{n} B_{m}\right) a_{k} \\
= & \alpha_{1}\left(\alpha_{2}+\prod_{k=3}^{n}\left[\alpha_{k}+{ }_{\ell=1}^{k-2} \frac{\left(-\beta_{k-\ell} \gamma_{k-\ell}\right)}{\alpha_{k-\ell}}\right]\right)-\beta_{1} \gamma_{1} \prod_{m=3}^{n}\left[\alpha_{m}+\prod_{\ell=1}^{m-2} \frac{\left(-\beta_{m-\ell} \gamma_{m-\ell}\right)}{\alpha_{m-\ell}}\right] \\
& -\sum_{k=3}^{n} \prod_{\ell=1}^{k-1} \beta_{\ell} \prod_{m=k+1}^{n}\left[\alpha_{m}+\prod_{\ell=1}^{m-2} \frac{\left(-\beta_{m-\ell} \gamma_{m-\ell}\right)}{\alpha_{m-\ell}}\right] \frac{\prod_{\ell=1}^{k-1} \gamma_{\ell}}{\prod_{\ell=2}^{k-1}\left[\alpha_{\ell}+K_{i=1}^{\ell-2} \frac{\left(-\beta_{\ell-i} \gamma_{\ell-i}\right)}{\alpha_{\ell-i}}\right]}
\end{aligned}
$$

which can be rearranged as 3.1 .

Making use of (2.1) and (2.2) yields 3.2 . The proof of Theorem 3.1 is complete. 


\section{Discussions}

In this section, we discuss our main results and related ones by several remarks.

Remark 4.1. In [3, p. 1018], it was stated that J. J. Sylvester found in 1854 that

$$
\left|M_{n}(s)\right|=\left|\begin{array}{ccccccccc}
s & 1 & 0 & 0 & \cdots & 0 & 0 & 0 & 0 \\
n & s & 2 & 0 & \cdots & 0 & 0 & 0 & 0 \\
0 & n-1 & s & 3 & \cdots & 0 & 0 & 0 & 0 \\
0 & 0 & n-2 & s & \cdots & 0 & 0 & 0 & 0 \\
\vdots & \vdots & \vdots & \vdots & \ddots & \vdots & \vdots & \vdots & \vdots \\
0 & 0 & 0 & 0 & \cdots & s & n-2 & 0 & 0 \\
0 & 0 & 0 & 0 & \cdots & 3 & s & n-1 & 0 \\
0 & 0 & 0 & 0 & \cdots & 0 & 2 & s & n \\
0 & 0 & 0 & 0 & \cdots & 0 & 0 & 1 & s
\end{array}\right|=\prod_{k=0}^{n}(s+n-2 k) .
$$

An application of (3.1) to $\left|M_{n}(s)\right|$ yields

$$
\begin{aligned}
\left|M_{n}(s)\right|= & s^{2}+(s-n) \prod_{m=3}^{n}\left[s+\prod_{\ell=1}^{m-2} \frac{-(m-\ell)(n-m+\ell+1)}{s}\right] \\
& -\sum_{k=3}^{n}\left[\prod_{\ell=1}^{k-1} \ell(n-\ell+1)\right] \frac{\prod_{m=k+1}^{n}\left[s+K_{\ell=1}^{m-2} \frac{-(m-\ell)(n-m+\ell+1)}{s}\right]}{\prod_{m=2}^{k-1}\left[s+K_{\ell=1}^{m-2} \frac{-(m-\ell)(n-m+\ell+1)}{s}\right]} \\
= & s^{2}+(s-n) \prod_{m=3}^{n}\left[s+\prod_{\ell=1}^{m-2} \frac{-(m-\ell)(n-m+\ell+1)}{s}\right] \\
& -n ! \sum_{k=3}^{n} \frac{(k-1) !}{(n-k+1) !} \frac{\prod_{m=k+1}^{n}\left[s+K_{\ell=1}^{m-2} \frac{-(m-\ell)(n-m+\ell+1)}{s}\right]}{\prod_{m=2}^{k-1}\left[s+K_{\ell=1}^{m-2} \frac{-(m-\ell)(n-m+\ell+1)}{s}\right]} \\
\triangleq & s^{2}+(s-n) \prod_{m=3}^{n} S(s ; m, n)-n ! \sum_{k=3}^{n} \frac{(k-1) !}{\prod_{m=k+1}^{n} S(s ; m, n)}
\end{aligned}
$$

Now we try to explicitly compute

$$
S(s ; m, n)=s+\stackrel{m-2}{K}_{\ell=1}^{-(m-\ell)(n-m+\ell+1)} .
$$

When $m=3$, it is easy to obtain that

$$
S(s ; 3, n)=\frac{s^{2}-2(n-1)}{s} \triangleq \frac{\beta_{1}}{\alpha_{1}} .
$$

When $m=4$, employing the above result for $S(s ; 3, n)$, we can acquire

$$
S(s ; 4, n)=\frac{\beta_{1} s-3(n-2) \alpha_{1}}{\beta_{1}}=\frac{s\left(s^{2}-5 n+8\right)}{s^{2}-2 n+2} \triangleq \frac{\beta_{2}}{\alpha_{2}} .
$$

If assuming $S(s ; k+1, n)=\frac{\beta_{k-1}}{\alpha_{k-1}}$, then, by mathematical induction, we have

$$
S(s ; k+2, m)=\frac{\beta_{k}}{\alpha_{k}}=\frac{\beta_{k-1} s-\alpha_{k-1}(k+1)(n-k)}{\beta_{k-1}} .
$$


Note that $\alpha_{k-1}=\beta_{k-2}$. Then

$$
\beta_{k}-\beta_{k-1} s+\beta_{k-2}(k+1)(n-k)=0 .
$$

Further replacing $k$ by $k+2$ results in

$$
\beta_{k+2}-\beta_{k+1} s+(k+3)(n-k-2) \beta_{k}=0 .
$$

By the approach utilized in [15, Theorem 3.1], the characteristic equation is

$$
t^{2}-s t+(k+3)(n-k-2)=0
$$

which has solutions

$$
t=\frac{s \pm \sqrt{s^{2}-4(k+3)(n-k-2)}}{2} .
$$

Consequently, it follows that

$$
\beta_{k}=A\left(\frac{s+\sqrt{s^{2}-4(k+3)(n-k-2)}}{2}\right)^{k-1}+B\left(\frac{s-\sqrt{s^{2}-4(k+3)(n-k-2)}}{2}\right)^{k-1},
$$

where

and

$$
A=-\frac{2 s^{3}-2(5 n-8)-\left(s^{2}-2 n+2\right)\left(s+\sqrt{s^{2}-20 n+80}\right)}{2 \sqrt{s^{2}-20 n+80}}
$$

$$
B=\frac{2 s^{3}-2(5 n-8)-\left(s^{2}-2 n+2\right)\left(s-\sqrt{s^{2}-20 n+80}\right)}{2 \sqrt{s^{2}-20 n+80}} .
$$

In a word, we provide an alternative expression for the Sylvester determinant $\left|M_{n}(s)\right|$.

Remark 4.2. In [3], by virtue of left eigenvector method, the determinants

$$
\begin{aligned}
\left|M_{n}(s, t)\right|= & \left|\begin{array}{cccccccc}
s & 1 & 0 & 0 & \cdots & 0 & 0 & 0 \\
n & s+t & 2 & 0 & \cdots & 0 & 0 & 0 \\
0 & n-1 & s+2 t & 3 & \cdots & 0 & 0 & 0 \\
0 & 0 & n-2 & s+3 t & \cdots & 0 & 0 & 0 \\
\vdots & \vdots & \vdots & \vdots & \ddots & \vdots & \vdots & \vdots \\
0 & 0 & 0 & 0 & \cdots & s+(n-2) t & n-1 & 0 \\
0 & 0 & 0 & 0 & \cdots & 2 & s+(n-1) t & n \\
0 & 0 & 0 & 0 & \cdots & 0 & 1 & s+n t
\end{array}\right| \\
& =\prod_{k=0}^{n}\left(s+\frac{n t}{2}+\frac{n-2 k}{2} \sqrt{t^{4}+4}\right)
\end{aligned}
$$


and

$$
\begin{aligned}
\left|M_{n}(s, t ; x, y)\right| & =\left|\begin{array}{cccccccc}
s & x & 0 & 0 & \cdots & 0 & 0 & 0 \\
n v & s+t & 2 x & 0 & \cdots & 0 & 0 & 0 \\
0 & (n-1) y & s+2 t & 3 x & \cdots & 0 & 0 & 0 \\
0 & 0 & (n-2) y & s+3 t & \cdots & 0 & 0 & 0 \\
\vdots & \vdots & \vdots & \vdots & \ddots & \vdots & \vdots & \vdots \\
0 & 0 & 0 & 0 & \cdots & s+(n-2) t & (n-1) x & 0 \\
0 & 0 & 0 & 0 & \cdots & 2 y & s+(n-1) t & n x \\
0 & 0 & 0 & 0 & \cdots & 0 & y & s+n t
\end{array}\right| \\
& =\prod_{k=0}^{n}\left(s+\frac{n t}{2}+\frac{n-2 k}{2} \sqrt{t^{4}+4 x y}\right)
\end{aligned}
$$

of tridiagonal matrices similar to the Sylvester matrix were collected and calculated. These evaluations can be computed alternatively by Theorem 3.1 .

Remark 4.3. The condition $b_{k} \neq 0$ for $2 \leq k \leq n$ in Theorem 2.1 is removed off in Theorem 2.2. Therefore, the explicit formula 2.3 is better than the recurrent formulas 2.1 and 2.2 .

Remark 4.4. The explicit formula 2.3. can be simply reformulated as

$$
\left|P_{n}\right|=\sum_{k=1}^{n}(-1)^{k+1}\left(\prod_{\ell=1}^{k-1} c_{\ell} \prod_{m=k+1}^{n} b_{m}\right) a_{k},
$$

where the empty product is understood to be 1 as usual.

\section{Remark 4.5. Let}

$$
U_{n}=\left(u_{i, j}\right)_{1 \leq i, j \leq n}=\left(\begin{array}{ccccccccc}
\alpha_{1} & \gamma_{1} & 0 & 0 & \cdots & 0 & 0 & 0 & \tau_{1} \\
\alpha_{2} & \beta_{2} & \gamma_{2} & 0 & \cdots & 0 & 0 & 0 & \tau_{2} \\
\alpha_{3} & 0 & \beta_{3} & \gamma_{3} & \cdots & 0 & 0 & 0 & \tau_{3} \\
\alpha_{4} & 0 & 0 & \beta_{4} & \cdots & 0 & 0 & 0 & \tau_{4} \\
\vdots & \vdots & \vdots & \vdots & \ddots & \vdots & \vdots & \vdots & \vdots \\
\alpha_{n-3} & 0 & 0 & 0 & \cdots & \beta_{n-3} & \gamma_{n-3} & 0 & \tau_{n-3} \\
\alpha_{n-2} & 0 & 0 & 0 & \cdots & 0 & \beta_{n-2} & \gamma_{n-2} & \tau_{n-2} \\
\alpha_{n-1} & 0 & 0 & 0 & \cdots & 0 & 0 & \beta_{n-1} & \gamma_{n-1} \\
\alpha_{n} & 0 & 0 & 0 & \cdots & 0 & 0 & 0 & \beta_{n}
\end{array}\right),
$$

where

$$
u_{i, j}= \begin{cases}\alpha_{i}, & 1 \leq i \leq n, j=1 \\ \beta_{i}, & 2 \leq i=j \leq n \\ \gamma_{i}, & 1 \leq i=j-1 \leq n-1 \\ \tau_{i}, & 1 \leq i \leq n-2, j=n \\ 0, & \text { otherwise. }\end{cases}
$$


The determinant $\left|U_{n}\right|$ can be rewritten as

$$
\begin{aligned}
& \left|U_{n}\right|=\left|\begin{array}{ccccccccc}
\alpha_{1} & \gamma_{1} & 0 & 0 & \ldots & 0 & 0 & 0 & \tau_{1} \\
\alpha_{2} & \beta_{2} & \gamma_{2} & 0 & \ldots & 0 & 0 & 0 & \tau_{2} \\
\alpha_{3} & 0 & \beta_{3} & \gamma_{3} & \ldots & 0 & 0 & 0 & \tau_{3} \\
\alpha_{4} & 0 & 0 & \beta_{4} & \ldots & 0 & 0 & 0 & \tau_{4} \\
\vdots & \vdots & \vdots & \vdots & \ddots & \vdots & \vdots & \vdots & \vdots \\
\alpha_{n-3} & 0 & 0 & 0 & \ldots & \beta_{n-3} & \gamma_{n-3} & 0 & \tau_{n-3} \\
\alpha_{n-2} & 0 & 0 & 0 & \ldots & 0 & \beta_{n-2} & \gamma_{n-2} & \tau_{n-2} \\
\alpha_{n-1} & 0 & 0 & 0 & \ldots & 0 & 0 & \beta_{n-1} & \gamma_{n-1} \\
\alpha_{n} & 0 & 0 & 0 & \ldots & 0 & 0 & 0 & \beta_{n}
\end{array}\right| \\
& =\left|\begin{array}{ccccccccc}
\alpha_{1}-\frac{\alpha_{n} \tau_{1}}{\beta_{n}} & \gamma_{1} & 0 & 0 & \cdots & 0 & 0 & 0 & 0 \\
\alpha_{2}-\frac{\alpha_{n} \tau_{2}}{\beta_{n}} & \beta_{2} & \gamma_{2} & 0 & \cdots & 0 & 0 & 0 & 0 \\
\alpha_{3}-\frac{\alpha_{n} \tau_{3}}{\beta_{n}} & 0 & \beta_{3} & \gamma_{3} & \cdots & 0 & 0 & 0 & 0 \\
\alpha_{4}-\frac{\alpha_{n} \tau_{4}}{\beta_{n}} & 0 & 0 & \beta_{4} & \cdots & 0 & 0 & 0 & 0 \\
\vdots & \vdots & \vdots & \vdots & \ddots & \vdots & \vdots & \vdots & \vdots \\
\alpha_{n-3}-\frac{\alpha_{n} \tau_{n-3}}{\beta_{n}} & 0 & 0 & 0 & \cdots & \beta_{n-3} & \gamma_{n-3} & 0 & 0 \\
\alpha_{n-2}-\frac{\alpha_{n} \tau_{n-2}}{\beta_{n}} & 0 & 0 & 0 & \cdots & 0 & \beta_{n-2} & \gamma_{n-2} & 0 \\
\alpha_{n-1}-\frac{\alpha_{n} \gamma_{n-1}}{\beta_{n}} & 0 & 0 & 0 & \cdots & 0 & 0 & \beta_{n-1} & 0 \\
\alpha_{n} & 0 & 0 & 0 & \cdots & 0 & 0 & 0 & \beta_{n}
\end{array}\right| \\
& =\beta_{n}\left|\begin{array}{cccccccc}
\alpha_{1}-\frac{\alpha_{n} \tau_{1}}{\beta_{n}} & \gamma_{1} & 0 & 0 & \cdots & 0 & 0 & 0 \\
\alpha_{2}-\frac{\alpha_{n} \tau_{2}}{\beta_{n}} & \beta_{2} & \gamma_{2} & 0 & \cdots & 0 & 0 & 0 \\
\alpha_{3}-\frac{\alpha_{n} \tau_{3}}{\beta_{n}} & 0 & \beta_{3} & \gamma_{3} & \cdots & 0 & 0 & 0 \\
\alpha_{4}-\frac{\alpha_{n} \tau_{4}}{\beta_{n}} & 0 & 0 & \beta_{4} & \cdots & 0 & 0 & 0 \\
\vdots & \vdots & \vdots & \vdots & \ddots & \vdots & \vdots & \vdots \\
\alpha_{n-3}-\frac{\alpha_{n} \tau_{n-3}}{\beta_{n}} & 0 & 0 & 0 & \cdots & \beta_{n-3} & \gamma_{n-3} & 0 \\
\alpha_{n-2}-\frac{\alpha_{n} \tau_{n-2}}{\beta_{n}} & 0 & 0 & 0 & \cdots & 0 & \beta_{n-2} & \gamma_{n-2} \\
\alpha_{n-1}-\frac{\alpha_{n} \gamma_{n-1}}{\beta_{n}} & 0 & 0 & 0 & \cdots & 0 & 0 & \beta_{n-1}
\end{array}\right| .
\end{aligned}
$$

An application of Theorem 2.1 and 2.2 and Remark 4.4 straightforwardly yields

$$
\left|U_{n}\right|=\sum_{k=1}^{n-2}(-1)^{k+1}\left(\alpha_{k} \beta_{n}-\tau_{k} \alpha_{n}\right) \prod_{\ell=1}^{k-1} \gamma_{\ell} \prod_{m=k+1}^{n-1} \beta_{m}+(-1)^{n}\left(\alpha_{n-1} \beta_{n}-\tau_{n-1} \alpha_{n}\right) \prod_{\ell=1}^{n-2} \gamma_{\ell}
$$

and

where

$$
\left|U_{n}\right|=\Lambda_{1, n-1} \prod_{k=2}^{n} \beta_{k}
$$

$$
\Lambda_{k, n-1}=\alpha_{k}-\frac{\alpha_{n}}{\beta_{n}} \tau_{k}-\frac{\gamma_{k}}{\beta_{k+1}} \Lambda_{k+1, n-1}, \quad 1 \leq k \leq n-2
$$


and $\Lambda_{n-1, n-1}=\alpha_{n-1}-\frac{\alpha_{n}}{\beta_{n}} \gamma_{n-1}$.

Remark 4.6. The determinant $\left|P_{n}\right|$ of $P_{n}$ in 1.2 can be rearranged as

$$
\begin{aligned}
& \left|P_{n}\right|=\left|\begin{array}{ccccccccc}
a_{1} & c_{1} & 0 & 0 & \cdots & 0 & 0 & 0 & 0 \\
a_{2} & b_{2} & c_{2} & 0 & \cdots & 0 & 0 & 0 & 0 \\
a_{3} & 0 & b_{3} & c_{3} & \cdots & 0 & 0 & 0 & 0 \\
a_{4} & 0 & 0 & b_{4} & \cdots & 0 & 0 & 0 & 0 \\
\vdots & \vdots & \vdots & \vdots & \ddots & \vdots & \vdots & \vdots & \vdots \\
a_{n-3} & 0 & 0 & 0 & \cdots & b_{n-3} & c_{n-3} & 0 & 0 \\
a_{n-2} & 0 & 0 & 0 & \cdots & 0 & b_{n-2} & c_{n-2} & 0 \\
a_{n-1} & 0 & 0 & 0 & \cdots & 0 & 0 & b_{n-1} & c_{n-1} \\
0 & 0 & 0 & 0 & \cdots & 0 & 0 & -\frac{a_{n} b_{n-1}}{a_{n-1}} & b_{n}-\frac{a_{n} c_{n-1}}{a_{n-1}}
\end{array}\right| \\
& =\cdots \\
& =\left|\begin{array}{ccccccc}
a_{1} & c_{1} & 0 & \ldots & 0 & 0 & 0 \\
0 & b_{2}-\frac{a_{2} c_{1}}{a_{1}} & c_{2} & \ldots & 0 & 0 & 0 \\
0 & -\frac{a_{3} b_{2}}{a_{2}} & b_{3}-\frac{a_{3} c_{2}}{a_{2}} & \ldots & 0 & 0 & 0 \\
0 & 0 & -\frac{a_{4} b_{3}}{a_{3}} & \ldots & 0 & 0 & 0 \\
\vdots & \vdots & \vdots & \ddots & \vdots & \vdots & \vdots \\
0 & 0 & 0 & \ldots & c_{n-3} & 0 & 0 \\
0 & 0 & 0 & \cdots & b_{n-2}-\frac{a_{n-2} c_{n-3}}{a_{n-3}} & c_{n-2} & 0 \\
0 & 0 & 0 & \cdots & -\frac{a_{n-1} b_{n-2}}{a_{n-2}} & b_{n-1}-\frac{a_{n-1} c_{n-2}}{a_{n-2}} & c_{n-1} \\
0 & 0 & 0 & \cdots & 0 & -\frac{a_{n} b_{n-1}}{a_{n-1}} & b_{n}-\frac{a_{n} c_{n-1}}{a_{n-1}}
\end{array}\right| \\
& =a_{1}\left|\begin{array}{cccccc}
b_{2}-\frac{a_{2} c_{1}}{a_{1}} & c_{2} & \ldots & 0 & 0 & 0 \\
-\frac{a_{3} b_{2}}{a_{2}} & b_{3}-\frac{a_{3} c_{2}}{a_{2}} & \ldots & 0 & 0 & 0 \\
0 & -\frac{a_{4} b_{3}}{a_{3}} & \ldots & 0 & 0 & 0 \\
\vdots & \vdots & \ddots & \vdots & \vdots & \vdots \\
0 & 0 & \ldots & c_{n-3} & 0 & 0 \\
0 & 0 & \cdots & b_{n-2}-\frac{a_{n-2} c_{n-3}}{a_{n-3}} & c_{n-2} & 0 \\
0 & 0 & \ldots & -\frac{a_{n-1} b_{n-2}}{a_{n-2}} & b_{n-1}-\frac{a_{n-1} c_{n-2}}{a_{n-2}} & c_{n-1} \\
0 & 0 & \ldots & 0 & -\frac{a_{n} b_{n-1}}{a_{n-1}} & b_{n}-\frac{a_{n} c_{n-1}}{a_{n-1}}
\end{array}\right| \\
& \triangleq a_{1}\left|Q_{n-1}\right| \text {. }
\end{aligned}
$$

Therefore, by virtue of Theorems 2.1 and 2.2 , we derive that the determinant $\left|Q_{n-1}\right|$ satisfies

$$
\left|Q_{n-1}\right|=\frac{\left|P_{n}\right|}{a_{1}}=\frac{\lambda_{1, n}}{a_{1}} \prod_{k=2}^{n} b_{k}
$$


and

$$
\left|Q_{n-1}\right|=\frac{\left|P_{n}\right|}{a_{1}}=\prod_{k=2}^{n} b_{k}-\frac{1}{a_{1}} \sum_{k=2}^{n}(-1)^{k}\left(\prod_{\ell=1}^{k-1} c_{\ell} \prod_{m=k+1}^{n} b_{m}\right) a_{k} .
$$

Further letting

in equations 4.1 and 4.2 reveals

$$
\begin{cases}\alpha_{k}=b_{k+1}-\frac{a_{k+1} c_{k}}{a_{k}}, & 1 \leq k \leq n-1 \\ \beta_{k}=c_{k+1}, & 1 \leq k \leq n-2 \\ \gamma_{k}=-\frac{a_{k+2} b_{k+1}}{a_{k+1}}, & 1 \leq k \leq n-2\end{cases}
$$

$$
\left|D_{n-1}\right|=\frac{\lambda_{1, n}}{a_{1}} \prod_{k=2}^{n} b_{k}
$$

and

$$
\left|D_{n-1}\right|=\prod_{k=2}^{n} b_{k}-\frac{1}{a_{1}} \sum_{k=2}^{n}(-1)^{k}\left(\prod_{\ell=1}^{k-1} c_{\ell} \prod_{m=k+1}^{n} b_{m}\right) a_{k} .
$$

From the second equality in (4.3), it is not difficult to see that $c_{k}=\beta_{k-1}$ for $2 \leq k \leq n-1$. If we can derive another relations from 4.3) to express $a_{k}$ for $1 \leq k \leq n$ and $b_{k}$ for $2 \leq k \leq n$ in terms of $\alpha_{k}$ for $1 \leq k \leq n-1, \beta_{k}$ for $1 \leq k \leq n-2$, and $\gamma_{k}$ for $1 \leq k \leq n-2$, then, by substituting these relations into 4.4 and (4.5), an alternative and explicit expression for evaluation of $\left|D_{n}\right|$ would be concluded. This is an open problem and we leave it to the interested readers.

Remark 4.7. In [1, Lemma 1.1] and [11, Lemma 2.1], it was acquired that

$$
\left|\begin{array}{cccccccc}
\tau_{1} & \tau_{2} & \tau_{3} & \tau_{4} & \cdots & \tau_{n-2} & \tau_{n-1} & \tau_{n} \\
\alpha & \beta & 0 & 0 & \cdots & 0 & 0 & 0 \\
\gamma & \alpha & \beta & 0 & \cdots & 0 & 0 & 0 \\
0 & \gamma & \alpha & \beta & \cdots & 0 & 0 & 0 \\
\vdots & \vdots & \vdots & \vdots & \ddots & \vdots & \vdots & \vdots \\
0 & 0 & 0 & 0 & \cdots & \beta & 0 & 0 \\
0 & 0 & 0 & 0 & \cdots & \alpha & \beta & 0 \\
0 & 0 & 0 & 0 & \cdots & \gamma & \alpha & \beta
\end{array}\right|=\sum_{k=1}^{n}(-1)^{k-1} \tau_{k} b^{n-k}(\beta \gamma)^{(k-1) / 2} U_{k-1}\left(\frac{\alpha}{2 \sqrt{\beta \gamma}}\right)
$$

where $U_{k}(s)$ is the $k$ th Chebyshev polynomials of the second kind, which can be generated 19 , 20, 22, by

$$
\frac{1}{1-2 s t+t^{2}}=\sum_{k=0}^{\infty} U_{k}(s) t^{k}, \quad|s|<1, \quad|t|<1 .
$$


Taking $\tau_{1}=\tau_{2}=\cdots=\tau_{n-1}=0$ and $\tau_{n}=1$ and reformulating, the formula 4.6 becomes

$$
\left|\begin{array}{cccccc}
\alpha & \beta & 0 & \cdots & 0 & 0 \\
\gamma & \alpha & \beta & \cdots & 0 & 0 \\
0 & \gamma & \alpha & \cdots & 0 & 0 \\
\vdots & \vdots & \vdots & \ddots & \vdots & \vdots \\
0 & 0 & 0 & \cdots & \alpha & \beta \\
0 & 0 & 0 & \cdots & \gamma & \alpha
\end{array}\right|_{n \times n}=(\beta \gamma)^{n / 2} U_{n}\left(\frac{\alpha}{2 \sqrt{\beta \gamma}}\right)
$$

This is different from

$$
\left|\begin{array}{cccccc}
\alpha & \beta & 0 & \cdots & 0 & 0 \\
\gamma & \alpha & \beta & \cdots & 0 & 0 \\
0 & \gamma & \alpha & \cdots & 0 & 0 \\
\vdots & \vdots & \vdots & \ddots & \vdots & \vdots \\
0 & 0 & 0 & \cdots & \alpha & \beta \\
0 & 0 & 0 & \cdots & \gamma & \alpha
\end{array}\right|_{n \times n}= \begin{cases}\frac{\left(\alpha+\sqrt{\alpha^{2}-4 \beta \gamma}\right)^{n+1}-\left(\alpha-\sqrt{\alpha^{2}-4 \beta \gamma}\right)^{n+1}}{(n+1)\left(\frac{\alpha}{2}\right)^{n},} & \alpha^{2} \neq 4 \beta \gamma \\
2^{n+1} \sqrt{\alpha^{2}-4 \beta \gamma} & \alpha^{2}=4 \beta \gamma\end{cases}
$$

and

$$
\left|\begin{array}{cccccc}
\alpha & \beta & 0 & \cdots & 0 & 0 \\
\gamma & \alpha & \beta & \cdots & 0 & 0 \\
0 & \gamma & \alpha & \cdots & 0 & 0 \\
\vdots & \vdots & \vdots & \ddots & \vdots & \vdots \\
0 & 0 & 0 & \cdots & \alpha & \beta \\
0 & 0 & 0 & \cdots & \gamma & \alpha
\end{array}\right|_{n \times n}=\prod_{j=1}^{n}\left(\beta+2 \alpha \sqrt{\frac{\gamma}{\alpha}} \cos \frac{j \pi}{n+1}\right)
$$

which are established and collected in [15, pp. 130] and [18, Theoem 4].

Comparing 4.7 with 4.8 and 4.9, taking $\beta=\gamma=1$ and $\alpha=2 s$, and simplifying yield

$$
\begin{aligned}
U_{n}(s) & =\prod_{j=1}^{n}\left(1+2 \sqrt{2 s} \cos \frac{j \pi}{n+1}\right) \\
& = \begin{cases}\frac{\left(s+\sqrt{s^{2}-1}\right)^{n+1}-\left(s-\sqrt{s^{2}-1}\right)^{n+1}}{2^{n+1} \sqrt{s^{2}-1}}, & s^{2} \neq 1 \\
(n+1) s^{n}, & s^{2}=1\end{cases}
\end{aligned}
$$

which are alternative explicit formulas for the Chebyshev polynomials of the second kind $U_{n}(s)$.

Remark 4.8. On 21 September 2019, we were reminded of the paper [10] in which an alternative explicit formula for elements of the inverse of a tridiagonal matrix and an efficient and fast computing method to obtain elements of the inverse of a tridiagonal matrix by backward continued fractions were investigated.

Remark 4.9. Theorem 2.2 in this paper has been applied in the proof of [17, Theorem 3.3].

Remark 4.10. This paper is a revised version of the preprint [21]. 
Acknowledgements. The authors appreciate anonymous referees for their careful corrections to and valuable comments on the original version of this paper.

Funding. The fourth author was supported by the National Research Foundation of Korea under Grant NRF-2018R1D1A1B07041846, South Korea.

Conflict of interest. No potential conflict of interest was reported by the authors.

\section{REFERENCES}

[1] D. Bozkurt, C. M. Da Fonseca, and F. Ylmaz, The determinants of circulant and skew-circulant matrices with tribonacci numbers, Math. Sci. Appl. E-Notes 2 (2014), no. 2, 67-75.

[2] Z. R. Chen, Inversion of general tridiagonal matrices, J. Numer. Methods Comput. Appl. 8 (1987), no. 3, 158-164. (Chinese)

[3] W. Chu, Fibonacci polynomials and Sylvester determinant of tridiagonal matrix, Appl. Math. Comput. 216 (2010), 1018-1023; available online at https://doi.org/10.1016/j.amc.2010.01.089

[4] D. F. G. Coelho, V. S. Dimitrov, and L. Rakai, Efficient computation of tridiagonal matrices largest eigenvalue, J. Comput. Appl. Math. 330 (2018), 268-275; available online at https://doi.org/10.1016/j.cam. 2017.08 .008

[5] M. El-Mikkawy and A. Karawia, Inversion of general tridiagonal matrices, Appl. Math. Lett. 19 (2006), no. 8, 712-720; available online at https://doi.org/10.1016/j.aml.2005.11.012.

[6] M. A. El-Shehawey, Gh. A. El-Shreef, and A. Sh. Al-Henawy, Analytical inversion of general periodic tridiagonal matrices, J. Math. Anal. Appl. 345 (2008), no. 1, 123-134; available online at https://doi.org/10. $1016 / j \cdot j$ maa.2008.04.002

[7] L. Euler, An essay on continued fractions, translated from the Latin by B. F. Wyman and M. F. Wyman, Math. Systems Theory 18 (1985), no. 4, 295-328; available online at https://doi.org/10.1007/BF01699475

[8] C. F. Fischer and R. A. Usmani, Properties of some tridiagonal matrices and their application to boundary value problems, SIAM J. Numer. Anal. 6 (1969), no. 1, 127-142; available online at https://doi.org/10. $1137 / 0706014$

[9] Y. Huang and W. F. McColl, Analytical inversion of general tridiagonal matrices, J. Phys. A 30 (1997), no. 22, 7919-7933; available online at https://doi.org/10.1088/0305-4470/30/22/026

[10] E. Kılıç, Explicit formula for the inverse of a tridiagonal matrix by backward continued fractions, Appl. Math. Comput. 197 (2008), no. 1, 345-357; available online at https://doi.org/10.1016/j.amc.2007.07.046

[11] E. Kurklar and F. Yllmaz, A general formula for determinants and inverses of r-circulant matrices with third order recurrences, Math. Sci. Appl. E-Notes 7 (2019), no. 1, 1-8.

[12] S. Kouachi, Eigenvalues and eigenvectors of some tridiagonal matrices with non-constant diagonal entries, Appl. Math. (Warsaw) 35 (2008), no. 1, 107-120; available online at https://doi.org/10.4064/am35-1-7

[13] S. Kouachi, Eigenvalues and eigenvectors of tridiagonal matrices, Electron. J. Linear Algebra 15 (2006), Article 8, 115-133; available online at https://doi.org/10.13001/1081-3810.1223

[14] G. Panti, Slow continued fractions, transducers, and the Serret theorem, J. Number Theory 185 (2018), 121-143; available online at https://doi.org/10.1016/j.jnt.2017.08.034

[15] F. Qi, V. Čerňanová, and Y. S. Semenov, Some tridiagonal determinants related to central Delannoy numbers, the Chebyshev polynomials, and the Fibonacci polynomials, Politehn. Univ. Bucharest Sci. Bull. Ser. A Appl. Math. Phys. 81 (2019), no. 1, 123-136.

[16] F. Qi, V. Čerňanová, X.-T. Shi, and B.-N. Guo, Some properties of central Delannoy numbers, J. Comput. Appl. Math. 328 (2018), 101-115; available online at https://doi.org/10.1016/j.cam.2017.07.013 
[17] F. Qi and C.-J. Huang, Computing sums in terms of beta, polygamma, and Gauss hypergeometric functions, Rev. R. Acad. Cienc. Exactas Fís. Nat. Ser. A Mat. RACSAM 114 (2020), Art. 191, 9 pages; available online at https://doi.org/10.1007/s13398-020-00927-y.

[18] F. Qi and A.-Q. Liu, Alternative proofs of some formulas for two tridiagonal determinants, Acta Univ. Sapientiae Math. 10 (2018), no. 2, 287-297; available online at https://doi.org/10.2478/ausm-2018-0022

[19] F. Qi, D.-W. Niu, and D. Lim, Notes on explicit and inversion formulas for the Chebyshev polynomials of the first two kinds, Miskolc Math. Notes 20 (2019), no. 2, 1129-1137; available online at https://doi.org/ 10.18514/MMN.2019.2976

[20] F. Qi, D.-W. Niu, D. Lim, and B.-N. Guo, Some properties and an application of multivariate exponential polynomials, Math. Methods Appl. Sci. 43 (2020), no. 6, 2967-2983; available online at https://doi.org/ $10.1002 / \mathrm{mma} .6095$

[21] F. Qi, W. Wang, D. Lim, and B.-N. Guo, Some formulas for determinants of tridiagonal matrices in terms of finite generalized continued fractions, HAL preprint (2019), available online at https://hal. archives-ouvertes.fr/hal-02372394v1

[22] F. Qi, Q. Zou, and B.-N. Guo, The inverse of a triangular matrix and several identities of the Catalan numbers, Appl. Anal. Discrete Math. 13 (2019), no. 2, 518-541; available online at https://doi.org/10. 2298/AADM190118018Q

(Qi) School of Mathematical Sciences, Tianjin Polytechnic University, Tianjin 300387, China; ColLege of Mathematics and Physics, Inner Mongolia University for Nationalities, Tongliao 028043, Inner Mongolia, China

Email address: qifeng618@gmail.com, qifeng618@hotmail.com, qifeng618@qq.com

$U R L:$ https://qifeng618.wordpress.com, https://orcid.org/0000-0001-6239-2968

(Wang) School of Mathematics and Statistics, Hefei Normal University, Hefei 230601, Anhui, China Email address: wwen2014@mail.ustc.edu.cn

$U R L$ : https://orcid.org/0000-0001-5562-4038

(Guo) School of Mathematics and Informatics, Henan Polytechnic University, Jiaozuo 454010, HENAN, China

Email address: bai.ni.guo@gmail.com, bai.ni.guo@hotmail.com

$U R L$ : https://orcid.org/0000-0001-6156-2590

(Lim) Department of Mathematics Education, Andong National University, Andong 36729, Republic OF Korea

Email address: dgrim84@gmail.com, dklim@andong.ac.kr

$U R L$ : http://orcid.org/0000-0002-0928-8480 